\title{
The prevalence of left ventricular thrombus among patients with low ejection fraction by trans-thoracic echocardiography
}

\author{
Nouradden Noman Aljaber ${ }^{1}$, Zohoor Ali Mattash², \\ Sultan Abdulwadoud Alshoabi ${ }^{3}$, Fahad Hassan Alhazmi ${ }^{4}$
}

\begin{abstract}
Background and objectives: Ejection fraction (EF) is a measurement of heart function that reflects the portion of pumped out blood from the filled left ventricle per each heartbeat. The current study aimed to investigate the prevalence of left ventricular thrombus in patients with $\mathrm{EF}$ lower than $35 \%$ by using Transthoracic Echocardiography (TTE).

Methods: In this prospective study, 82 cardiac patients underwent TTE procedure in order to assess the presence of left ventricular thrombus (LVT) from January $1^{\text {st }}$ to December 31st 2017 at the Military Cardiac Centre in Sana'a, Yemen.

Results: Out of 82 patients enrolled in this study, the mean age was $49.13 \pm 14.8$ years and $87.8 \%$ were male. The mean of EF was $31.16 \%$ and LVT was found in $6.1 \%$. The spontaneous contrast was seen in $25.6 \%$ of patients indicating strong relationship with low EF $(p<0.001)$. Among patients with low EF, ischemic heart disease (IHD) was identified in $50 \%$, hypertension in $30.5 \%$, diabetes mellitus (DM) type 2 in $23.2 \%$, and hyperlipidemia $12.2 \%$. Exactly $80 \%$ of LVT were detected in IHD patients with dilated cardiomyopathy (DCMP) and $80 \%$ of detected LVT were apical in site.

Conclusion: Cardiac patients with low ejection fraction developed left ventricular thrombosis, and most of the affected patients were ischemic heart disease with dilated cardiomyopathy. Interestingly, spontaneous contrast was found high significantly in these patients, which may reflect the continuous process of thrombus formation.
\end{abstract}

KEYWORDS: Echocardiography, Ejection Fraction, Left Ventricular Thrombus, Spontaneous contrast.

\section{Abbreviations:}

EF: ejection fraction, ASE: American society of echocardiography, EACVI: European association of cardiovascular imaging, LV: left ventricle, LVEF: left ventricular ejection fraction, HFpEF: heart failure with preserved ejection fraction, HFrEF: heart failure with reduced ejection fraction, MI: myocardial infarction, DCMP: dilated cardiomyopathy, AF: atrial fibrillation, TTE: Transthoracic echocardiography, TEE: trans-esophageal echocardiography, RHD: rheumatic heart disease, HTN: hypertension, DM: diabetes mellitus, CHF: congestive heart failure, JVP: jugular venous pressure, CBC: complete blood count, LFT: liver function tests, RFT: renal function test, LVT: left ventricular thrombus, ECG: electrocardiography, LVT: left ventricular thrombus, EDV: end diastolic volume, ESV: end systolic volume, SPSS: statistical package for the social sciences, IBM: international business machines, SD: standard deviation.

How to cite this:

doi: https://doi.org/10.12669/pjms.36.4.1972

Aljaber NN, Mattash ZA, Alshoabi SA, Alhazmi FA. The prevalence of left ventricular thrombus among patients with low ejection fraction by trans-thoracic echocardiography. Pak J Med Sci. 2020;36(4):673-677. doi: https://doi.org/10.12669/pjms.36.4.1972

This is an Open Access article distributed under the terms of the Creative Commons Attribution License (http://creativecommons.org/licenses/by/3.0), which permits unrestricted use, distribution, and reproduction in any medium, provided the original work is properly cited.

Correspondence:

Sultan Abdulwadoud Alshoabi, MD, Email: alshoabisultan@yahoo.com

* Received for Publication:

* Edited and Corrected:

* Accepted for Publication:
November 17, 2019

February 5, 2020

February 17, 2020

\section{INTRODUCTION}

Ejection fraction (EF) is the portion of pumped out oxygen-rich blood from a filled left ventricle to the aorta per each heartbeat. It measures the pumping ability and function of the heart. The 
cutoff of normal EF value is poorly-defined, however $50 \%$ of volume of blood in the left ventricle $(\mathrm{LV})$ is considered as the lower boundary of normal. ${ }^{1}$ The American Society of Echocardiography (ASE) and the European Association of Cardiovascular Imaging (EACVI) consider the lowest limits of EF in male and female as $52 \%$ and $54 \%$ respectively. ${ }^{2}$ Left ventricular ejection fraction (LVEF) is a significant parameter for LV systolic function, which can be utilized as an independent predictor for mortality events after acute ischemic stroke. ${ }^{3}$ Based on the functional statues of the heart, heart failure (HF) is classified into: HF with preserved EF (HFpEF) and HF with reduced EF (HFrEF) that are occurred when EF is equal to or more than $50 \%$ and less than $40 \%$ respectively. ${ }^{4,5} \mathrm{LV}$ thrombus is a serious complication of severe LV systolic dysfunction. The pathophysiology of the LV thrombus is related to endothelial injury, hypercoagulable state, and stasis of blood flow secondary to myocardial infarction (MI), chronic heart failure, and dilated cardiomyopathy (DCMP). ${ }^{6} \mathrm{LV}$ apical thrombus is associate with low EF (35\%) and also with apical aneurysm. ${ }^{7}$ The heart is a significant source of systemic emboli, and the most common cardiogenic source of emboli are atrial fibrillation (AF), LV thrombus, and prosthetic valves. ${ }^{8} \mathrm{LV}$ apical thrombus is a serious complication of systolic dysfunction in patients with acute MI that is a risk of systemic embolization. ${ }^{9} \mathrm{EF}$ is measured by assessing the changes in lumen of the LV. ${ }^{10}$ Transthoracic echocardiography (TTE), and/or trans-esophageal echocardiography (TEE) plays an essential role in evaluation, diagnosis and plan management of patients with low EF. ${ }^{8} \mathrm{TTE}$ has $100 \%$ specificity for detecting LV thrombus. ${ }^{11}$

This study is intended firstly to assess the incidence of LV thrombus in patients with low $\mathrm{EF}$, and secondary to assess the relationship between common habits in Yemeni population such as khat chewing, smoking, shamma using with reduced EF of the heart. Khat is a plant that is chewed for its stimulant action which is popular in Yemen. ${ }^{12}$

Shammah is a snuff of smokeless tobacco that is used in the middle east especially Saudi arabia, Sudan and Yemen. ${ }^{13}$ In this study, we used TTE as it is the most common imaging technique used to assess heart diseases. Based on our knowledge, this is the first study intended to assess the prevalence of LV thrombus in low EF patients.

\section{METHODS}

Patient selection: This cross-sectional, prospective study was conducted at Military Cardiac Center in Sana'a, Yemen. Data of 82 patients who underwent echocardiography in the outpatient clinic from January $1^{\text {st }}$ to December 31st 2017 and met the inclusion criteria of this study were collected. The inclusion criteria were:

1. Patients with EF less than $35 \%$ and more than 12 years old age.

\section{The Exclusion criteria were:}

1. Patients with rheumatic heart disease (RHD) who were under anticoagulant drugs.

2. Patients with atrial fibrillation (AF).

Ethical approval: This study was approved by the Cardiac Center Research ethics committee. Full institutional ethical approval was obtained before beginning the study. Verbal consent from each patient was also obtained before examination. Confidentiality of all patient information was assured.

Variables assessed: A pre-tested questionnaire was prepared to include all relevant information. Researcher through direct interview filled the questionnaire with the patients. Explanation of the study purpose was explained to each patient, and consent was obtained. The following variables are included: sociodemographic, clinical history for cardiac disorders causes, risk factors such as hypertension (HTN), diabetes mellitus (DM), smoking, hyperlipidemia, drug in-use and disease duration, severity and complications.

Physical examination was performed for each patient looking for signs of congestive heart failure $(\mathrm{CHF})$ as increase jugular venous pressure (JVP), hepatomegaly, lower limb edema. Investigations were done included complete blood count (CBC), cardiac enzymes, liver function tests (LFT), renal function test (RFT), electrocardiogram (ECG), and chest $x$-ray (CXR). Patients with EF less than 35\% underwent another TTE to assess the presence of left ventricular thrombus (LVT).

Echocardiography (ECHO) procedure: The ECHO procedure was performed with the same ultrasound imaging machine (Philips I E 33, Australia) using a standard protocol. All selected patients were examined by the same cardiologist with 25 years' experience in echocardiography.

LVT was defined as a hyperechoic mass with definite margins adjacent to the myocardium on multiple plane views through the cardiac cycle. Regional wall motion abnormalities 

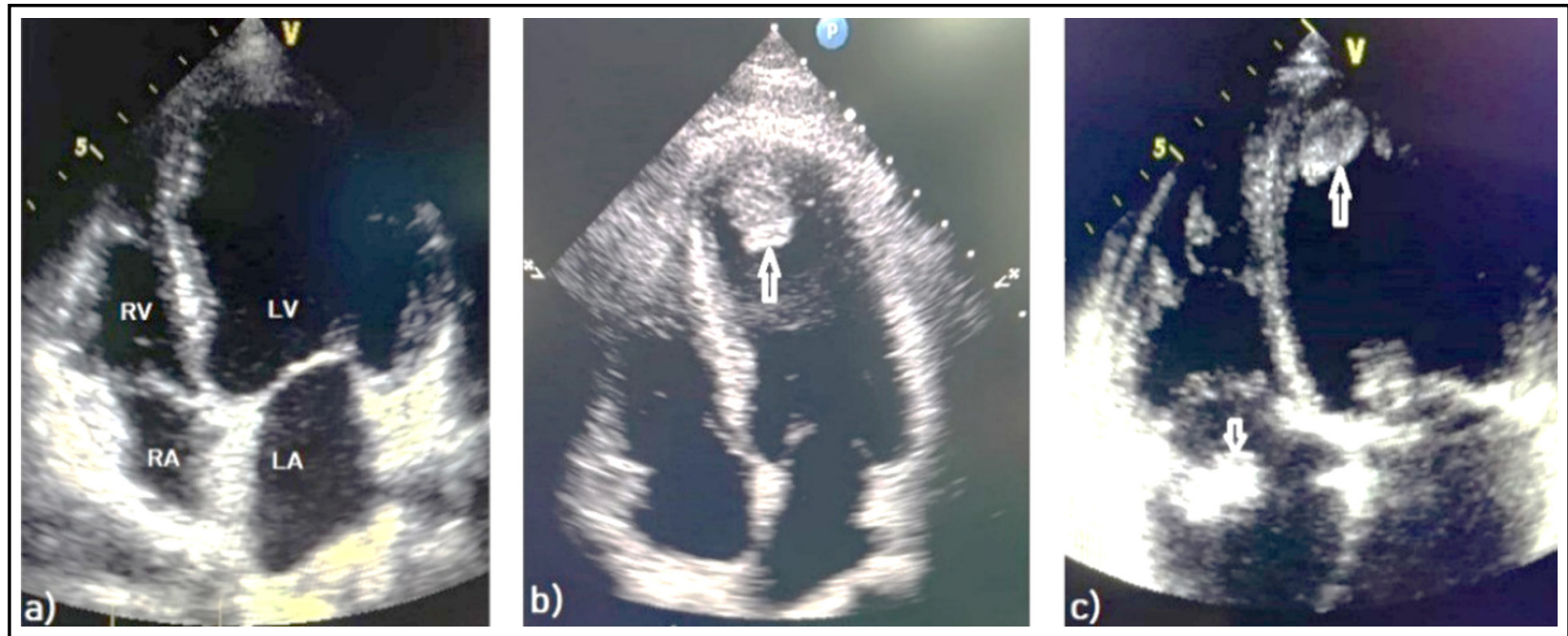

Fig.1: Transthoracic echocardiography images shows a) normal all cardiac chambers b) left ventricular thrombus (arrow), and c) left ventricular thrombus (long arrow) and right atrial thrombus (short arrow).

$\mathrm{LV}$, left ventricle; RV, right ventricle; LA, left atrium; RA, right atrium.

were considered present whether hypokinesia, akinesia or dyskinesia were observed in at least two segments of the left ventricular wall. Left ventricular function was assessed by echocardiographic examination. LV end diastolic and end-systolic volumes and ejection fraction were determined from apical 2 and 4 chamber views using Simpson`s biplane formula according to recommendation of the American Society of echocardiography: $\mathrm{EF}=(\mathrm{EDV}-\mathrm{ESV}) / \mathrm{EDV} .^{2}$

EF: Ejection fraction

EDV: End Diastolic Volume (ml)

ESV: End Systolic Volume (ml)

Statistical analysis: Data analysis was performed using SPSS version 21 (IBM). Data are presented as frequency and percentage for continuous variables and mean \pm Standard Deviation (SD) for descriptive variables. Quantitative data were analysed using One sample student t-test, and qualitative data were compared using Chi square test. P-value was assumed to be significant when $\mathrm{P}<0.05$.

Table-I: Shows findings of TTE in patients with $<35 \% \mathrm{EF}$.

\begin{tabular}{lcc}
\hline Patients with EF less than 35\% & Frequency & $\%$ \\
\hline EF $<30$ & 21 & 25.6 \\
EF 30-35 & 61 & 74.4 \\
\hline Patients with positive findings: & Frequency & $\%$ \\
\hline LVT & 5 & 6.1 \\
Spontaneous contrast & 21 & 25.6 \\
\hline
\end{tabular}

Table-II: Shows the comorbidities of the patients with $<35 \% \mathrm{EF}$.

\begin{tabular}{lccc}
\hline Comorbidities & Frequency & $\%$ & -value \\
\hline Ischemic heart disease (IHD) & 41 & 50 & 0.07 \\
Hypertension (HTN) & 25 & 30.5 & 0.21 \\
DM type-2 & 19 & 23.2 & 0.65 \\
Hyperlipedemia & 10 & 12.2 & 0.98 \\
\hline
\end{tabular}

\section{RESULTS}

In total, 82 patients who had $\mathrm{EF}<35 \%$ included in this study. The mean age at diagnosis was $49.13 \pm 14.8$ years; $87.8 \%$ were male and $12.2 \%$ were female. Echocardiographic findings showed the mean of EF was $31.16 \%$ and LVT was present in $6.1 \%$, which is statistically not significant $(p=$ 0.61 ). The spontaneous contrast picture was seen in $25.6 \%$ of patients $(\mathrm{p}<0.001)-($ Table-I). Among

Table-III: Shows the common bad habits of the patients.

\begin{tabular}{lcc}
\hline Habits & Frequency & $\%$ \\
\hline Smokers & 2 & 2.4 \\
Khat chewers & 23 & 28 \\
Khat chewers + Smokers & 29 & 35.4 \\
Khat chewers + Smokers & 10 & 12.2 \\
$\quad$ + Shammah users & & \\
Khat chewers + Smokers + Alcoholic & 1 & 1.2 \\
Khat chewers + Shammah users & 3 & 3.7 \\
None & 14 & 17 \\
\hline Total & 82 & 100 \\
\hline
\end{tabular}


Nouradden Noman Aljaber et al.

Table-IV: Details of thrombus among the 5 patients of LVT in the patients of $<35 \% \mathrm{EF}$.

\begin{tabular}{cllclll}
\hline No. & Site & Nature & Size $(\mathrm{mm})$ & EF & Disease & Co-morbidities \\
\hline 1 & Apical & Organized & N/A & 29 & DCMP & IHD + $\uparrow$ lipid \\
2 & Apical & Organized & N/A & 29 & DCMP & IHD \\
3 & Apical & Organized & $7 \times 12$ & 34 & DCMP & IHD + DM \\
4 & Apical & Organized & $12 \times 8$ & 32 & DCMP & IHD + DM+ HHD \\
5 & Mural & Organized & $14 \times 40$ & 25 & HHD & HHD \\
\hline
\end{tabular}

DCMP: Dilated cardiomyopathy, HHD: Hypertensive heart disease,

IHD: Ischemic heart disease, DM: Diabetes mellitus.

patients with low EF, IHD was identified in $50 \%$, hypertension in $30.5 \%$, DM type 2 in $23.2 \%$, and hyperlipidemia $12.2 \%$ (Table-II).

Khat chewer and smokers were the most common popular habits in the patients of low EF then Khat chewing only but no significant relationship $(\mathrm{p}=0.96)$ (Table-III). Exactly $80 \%$ of LVT were apical, $80 \%$ were in ischemic heart disease patients with dilated cardiomyopathy (Table-IV).

\section{DISCUSSION}

$\mathrm{LV}$ thrombus is a serious complication of heart failure. In this study, we assessed the prevalence of LV thrombus in patients with heart failure with low EF. Disertori et al., reported that patients with LVEF less than 35\% have a high risk of death due to evolution of HF. ${ }^{14}$ In this study, we found LV thrombus in $6.1 \%$ of patients and that may be a source of serious emboli to any other critical organ including the brain. This result is consistent with the results of Watanabe et al., who reported significant relationship between cerebral micro bleeds and low left ventricular EF. ${ }^{15}$ In this study, $50 \%$ of patients with low EF was associate with IHD. This result is consistent with Vedin et al., who reported the high prevalence of IHD in patients with $\mathrm{HFrEF}$ with increased risk of new IHD events. ${ }^{16}$ The result are also consistent with Rahmayani et al., who reported that low left ventricular EF affects the clinical outcomes of IHD patients. ${ }^{17}$ In this study, we found that $80 \%$ of LV thrombus were apical. This result is consistent with Adar et al., who reported that LV thrombus often formed in the LV apex. ${ }^{18}$ The result also consistent with Kaolawanich et al., who reported that apical area index is a new index in patients with LV systolic dysfunction to predict LV thrombus. ${ }^{19}$ The apical site of thrombi was explained by Benito who reported that the apex of the ventricle is the most prone to blood stasis. ${ }^{20}$ In this study, $80 \%$ of the patients with low
EF were khat chewers and $80 \%$ of LV thrombus were in IHD. This result is consistent with AlMotarreb et al., who reported that khat chewing is a risk factor for acute MI by causing coronary spasm. Mega et al., reported that heavy khat chewers are at higher risk factor of acute MI than moderate khat chewers. ${ }^{21,22}$

Limitations of the study: It was a single-center study and the sample size was limited by 82 patients.

\section{CONCLUSION}

Cardiac patients with low ejection fraction developed left ventricular thrombosis and most of the affected patients were of dilated cardiomyopathy. Interestingly, spontaneous contrast was significantly high in patients with low ejection fraction which is a continuous process of thrombus formation. This make starting anticoagulant therapy in this group of cardiac patients is an individual decision based on patient and physician discussion.

\section{Conflicts of interest: None.}

Financial support: None.

\section{REFERENCES}

1. Lund LH. Heart Failure with Mid-Range Ejection Fraction: Lessons from CHARM. Card Fail Rev. 2018;4(2):70-72. doi: 10.15420/cfr.2018.11.2

2. Lang RM, Badano LP, Mor-Avi V, Afilalo J, Armstrong A, Ernande L, et al. Recommendations for cardiac chamber quantification by echocardiography in adults: an update from the American Society of Echocardiography and the European Association of Cardiovascular Imaging. Eur Heart J Cardiovasc Imaging. 2015;16:233-70. doi: 10.1093/ehjci/jev014

3. Lee JY, Sunwoo JS, Kwon KY, Roh H, Ahn MY, Lee $\mathrm{MH}$, et al. Left Ventricular Ejection Fraction Predicts Poststroke Cardiovascular Events and Mortality in Patients without Atrial Fibrillation and Coronary Heart Disease. Korean Circ J. 2018;48(12):1148-1156. doi: 10.4070/kcj.2018.0115

4. Inamdar AA, Inamdar AC. Heart Failure: Diagnosis, Management and Utilization. J Clin Med. 2016;5(7):62. doi: $10.3390 /$ jcm5070062 
5. Mentzer G, Hsich EM. Heart Failure with Reduced Ejection Fraction in Women: Epidemiology, Outcomes, and Treatment. Heart Fail Clin. 2019;15(1):19-27. doi: 10.1016/j.hfc.2018.08.003

6. Satish M, Vukka N, Apala D, Mahfood Haddad T, Gupta J. Left Ventricular Thrombus After Acute Decompensated Heart Failure in the Setting of Ischemic Cardiomyopathy. Cureus. 2019;11(4):e4537. doi: 10.7759/cureus.4537

7. Oh JK, Park JH, Lee JH, Kim J, Seong IW. Shape and Mobility of a Left Ventricular Thrombus Are Predictors of Thrombus Resolution. Korean Circ J. 2019;49(9):829-837. doi: 10.4070/ kcj.2018.0346

8. Celeste F, Muratori M, Mapelli M, Pepi M. The Evolving Role and Use of Echocardiography in the Evaluation of Cardiac Source of Embolism. J Cardiovasc Echogr. 2017;27(2):33-44. doi: 10.4103/jcecho.jcecho_1_17

9. Choi UL, Park JH, Sun BJ, Oh JK, Seong SW, Lee JH. Impaired left ventricular diastolic function is related to the formation of left ventricular apical thrombus in patients with acute anterior myocardial infarction. Heart Vessels. 2018;33(5):447-452. doi: 10.1007/s00380-017-1079-z

10. MacIver DH, Adeniran I, Zhang H. Left ventricular ejection fraction is determined by both global myocardial strain and wall thickness. Int J Cardiol Heart Vasc. 2015;7:113-118. doi: 10.1016/j.ijcha.2015.03.007

11. Schubmehl HB, Tchantchaleishvili V, Storoznsky E, Alexis JD, Chen L, Massey HT. Echocardiographic detection of left ventricular thrombus in patients undergoing Heart Mate II left ventricular assist device implantation. Int J Artif Organs. 2016;39(9):491-496. doi: 10.5301/ijao.5000526

12. El-Menyar A, Mekkodathil A, Al-Thani H, Al-Motarreb A. Khat use: history and heart failure. Oman Med J. 2015;30(2):77-82. doi: 10.5001/omj.2015.18

13. Al-Hebshi NN, Li S, Nasher AT, El-Setouhy M, Alsanosi $\mathrm{R}$, Blancato J, et al. Exome sequencing of oral squamous cell carcinoma in users of Arabian snuff reveals novel candidates for driver genes. Int J Cancer. 2016;139(2):363372. doi: 10.1002/ijc.30068

14. Disertori M, Gulizia MM, Casolo G, Delise P, Di Lenarda A, Di Tano G, et al. Improving the appropriateness of sudden arrhythmic death primary prevention by implantable cardioverter-defibrillator therapy in patients with low left ventricular ejection fraction. Point of view. J Cardiovasc Med (Hagerstown). 2016;17(4):245-255. doi: 10.2459/ JCM.0000000000000368

15. Watanabe T, Kanzaki Y, Yamauchi Y, Ito T, Nishida Y, Yamamura K. Increased prevalence of cerebral microbleeds in patients with low left ventricular systolic function. Heart Vessels. 2019. doi: 10.1007/s00380-019-01503-0
16. Vedin O, Lam CSP, Koh AS, Benson L, Teng THK, Tay WT. Significance of Ischemic Heart Disease in Patients with Heart Failure and Preserved, Midrange, and Reduced Ejection Fraction: A Nationwide Cohort Study. Circ Heart Fail. 2017;10(6) pii:e003875. doi: 10.1161/CIRCHEARTFAILURE.117.003875

17. Rahmayani F, Paryono, Setyopranoto I. The Role of Ejection Fraction to Clinical Outcome of Acute Ischemic Stroke Patients. J Neurosci Rural Pract. 2018;9(2):197-202. doi: 10.4103/jnrp.jnrp_490_17

18. Adar A, Onalan O, Cakan F. Newly developed left ventricular apical thrombus under dabigatran treatment. Blood Coagul Fibrinolysis. 2018;29(1):126-128. doi: 10.1097/ MBC.0000000000000671

19. Kaolawanich $Y$, Boonyasirinant $T$. Usefulness of apical area index to predict left ventricular thrombus in patients with systolic dysfunction: a novel index from cardiac magnetic resonance. BMC Cardiovasc Disord. 2019;19(1):15. doi: 10.1186/s12872-018-0988-9

20. Benito Y, Martinez-Legazpi P, Rossini L, et al. AgeDependence of Flow Homeostasis in the Left Ventricle. Front Physiol. 2019; 10:485. doi:10.3389/fphys.2019.00485

21. Al-Motarreb A, Shabana A, El-Menyar A. Epicardial coronary arteries in khat chewers presenting with myocardial infarction. Int J Vasc Med. 2013;2013:857019. doi: $10.1155 / 2013 / 857019$

22. Mega TA, Dabe NE. Khat (Catha Edulis) as a Risk Factor for Cardiovascular Disorders: Systematic Review and Meta-Analysis. Open Cardiovasc Med J. 2017;11:146-155. doi: 10.2174/1874192401711010146

\section{Author`s Contribution:}

NMA: Conceptualization, and data collection.

ZAM: data analysis.

SAA: writing the original draft and is responsible for integrity of research.

FHA: manuscript review \& editing.
1. Nouradden Noman Aljaber, MD

Faculty of Medicine,

Sana'a University, Sana'a, Republic of Yemen.

2. Zohoor Ali Mattash, MD

Military Cardiac Center

Sana'a, Republic of Yemen.

3. Sultan Abdulwadoud Alshoabi, MD

4. Fahad Hassan Alhazmi, PhD

3,4: Department of Diagnostic Radiology Technology, College of Applied Medical Sciences, Taibah University, Kingdom of Saudi Arabia.

Correspondence:

Sultan Abdulwadoud Alshoabi, MD, Associate Professor of Radiology,

Department of Diagnostic Radiology Technology, College of Applied Medical Sciences, Taibah University, Kingdom of Saudi Arabia.

Email: alshoabisultan@yahoo.com

* Received for Publication:

November 17, 2019

* Edited and Corrected:

* Accepted for Publication:
February 5, 2020

February 17, 2020

$\begin{array}{lllll}\text { Pak J Med Sci } \quad \text { May - June } 2020 & \text { Vol. } 36 & \text { No. } 4 & \text { www.pjms.org.pk } 677\end{array}$ 